\title{
Aspectos epidemiológicos da leishmaniose visceral canina no município de Pedro Leopoldo, Minas Gerais, 2003
}

\author{
[Epidemiologic aspects of canine visceral leishmaniasis in Pedro Leopoldo district, \\ Minas Gerais, 2003] \\ L.A.B. Naveda ${ }^{1}$, E.C. Moreira ${ }^{2 *}$, J.G. Machado, J.R.C. Moraes ${ }^{2}$, A.P. Marcelino ${ }^{1}$ \\ ${ }^{1}$ Alunos de pós-graduação - EV-UFMG - Belo Horizonte, MG \\ ${ }^{2}$ Escola de Veterinária - UFMG \\ Caixa Postal 567 \\ 30123-970 - Belo Horizonte, MG
}

\begin{abstract}
RESUMO
Determinou-se a prevalência da leishmaniose visceral canina (LVC) no município de Pedro Leopoldo, MG, 2003, por meio de inquérito soro-epidemiológico. Coletaram-se amostras de sangue de 2.185 cães e aplicou-se questionário semi-estruturado em cada residência. Os animais foram divididos em quatro grupos, sendo o grupo A constituído de 1240 cães das áreas urbana e rural; o grupo B de 705 cães de áreas em torno dos focos; o grupo $\mathrm{C}$, de 134 cães, cujas amostras foram coletadas durante o dia da vacinação anti-rábica oficial; e o grupo D composto de 111 cães errantes. Os dados foram armazenados em banco de dados EPIINFO 6.04b. Os 36 cães positivos foram localizados espacialmente com auxílio de GPS (Global Positioning System), e o georreferenciamento realizado por meio do programa AUTOCAD 2000. A prevalência global da LVC foi de $1,4 \%$ e variou de $1,1 \%$ na área urbana e $4,2 \%$ na rural. Estes resultados permitiram concluir que Pedro Leopoldo, de acordo com a classificação oficial do Ministério da Saúde, é área silenciosa, receptiva e vulnerável para LVC. A distribuição espacial dos casos é diferenciada e requer medidas sanitárias de acordo com cada área de risco.

Palavras-chave: cão, leishmaniose visceral, epidemiologia
\end{abstract}

\begin{abstract}
The prevalence of canine visceral leishmaniasis (CVL) in Pedro Leopoldo district, 2003 was evaluated. A serum-epidemic inquiry was accomplished by collecting blood samples of dogs and by applying a semi structured questionnaire, according to groups, in each visited residence: group A, constituted of 1240 dogs of the urban and rural areas; group B, constituted of 705 dogs sampled around the focuses; group $C$, constituted of 134 dogs, wich samples were collected during the caninelfeline antirabic vaccination; and group D, constituted of 111 wandering dogs. The data were stored in the EPI INFO 6.04b database. Positive dogs were spatially located using GPS (Global Positioning System) and the geographycal reference was made through the AUTOCAD 2000 program. The district global prevalence for canine visceral leishmaniasis was of $1.4 \%$, varying from $1.1 \%$ to $4.2 \%$. These results allowed to conclude that Pedro Leopoldo is a silent area, receptive and vulnerable to CVL, in agreement with the Ministry of Health official classification. The spacial distribution of CVL is not homogeneous and it requires sanitary measures according to each risk area. The obtained knowledge allows to establish effective strategies for the control of CVL in that distric.
\end{abstract}

Keywords: dog, visceral leishmaniasis, epidemiology

Recebido em 15 de março de 2005

Aceito em 15 de maio de 2006

*Autor para correspondência (corresponding author)

E-mail: elviocm@vet.ufmg.br 


\section{INTRODUÇÃO}

A leishmaniose visceral (LV) representa hoje uma das principais enfermidades que afetam a população canina e a humana. No Brasil, é doença endêmica, e a transmissão já foi descrita em vários estados, com exceção os da região Sul. Está registrada em 19 das 27 unidades da Federação, com aproximadamente 1600 municípios que apresentam transmissão autóctone. A maior incidência encontra-se na região Nordeste com $65 \%$ do total de casos, seguida da região Sudeste (14\%), Norte (14\%) e Centro-Oeste (7\%) (Manual..., 2003; Brasil, 2004). Em Minas Gerais, de 2001 até julho de 2004, $127(14,9 \%)$ dos municípios que a compõem possuíam casos humanos confirmados, totalizando 1128 confirmações. A incidência variou de 1,13 a 1,58/100.000 casos/habitante (Minas Gerais, 2004).

De caráter inicialmente rural, atualmente ela se encontra urbanizada e instalada em grandes centros populacionais. A mudança do perfil epidemiológico da leishmaniose para áreas urbanas torna-se visível principalmente nas grandes periferias onde $\mathrm{o}$ adensamento populacional humano e canino é alto, o processo da verticalização das cidades é constante e o estado imunológico da população é alterado por fatores como estresse, desnutrição, drogas, enfermidades transmissíveis, entre elas, o HIV.

O controle da doença, proposto pelo Ministério da Saúde (Manual... 2003), deve ser feito de forma integrada e sob a responsabilidade do SUS. Está centrado no diagnóstico e tratamento precoce dos casos humanos, redução da população de flebotomíneos, eliminação dos reservatórios e atividades de educação em saúde.

No município de Pedro Leopoldo, até 2002, os dados sobre leishmaniose visceral canina (LVC) e humana eram escassos e dispersos. Haviam sido confirmados dois casos humanos, um em 1999 e outro em 2002. Esses casos foram classificados como importados pela investigação epidemiológica. Os diagnósticos caninos constavam de esporádicas notificações de cães atendidos em clínicas particulares locais e do inquérito amostral realizado em 1992, pela Fundação Nacional de Saúde, quando foi encontrado somente um animal positivo $(0,2 \%)$ de 524 cães examinados (Cabral, 1995). A presença de flebotomíneos Lutzomyia (Lutzomyia) longipalpis (Lutz e Neiva, 1912) no município foi confirmada por trabalhos feitos por Martins et al. (1978).

A proximidade de Pedro Leopoldo de cidades onde a LV é endêmica, associada às condições climáticas e fisiográficas propícias à manutenção da doença e com a falta de controle quanto ao trânsito de cães de outras localidades, representam fatores que contribuem para o risco de introdução e difusão da LV no município.

Atualmente, independente da condição sócioeconômica de cada país, as ações e os programas de controle de doenças são definidos de acordo com as áreas de risco (Manual...,2003). Torna-se cada vez mais necessário, face à grande demanda de recursos financeiros para suprir as carências sociais, que o uso de determinada alternativa de controle seja eficaz e economicamente viável. $\mathrm{O}$ controle da LV deve ser feito de maneira estratégica, identificando áreas de risco e, para isso, alternativas como o inquérito sorológico canino, baseado em critérios epidemiológicos, são metodologias ainda indicadas para o seu combate e vigilância (Brenner, 1957).

O objetivo deste trabalho foi estimar a prevalência da leishmaniose visceral canina no município de Pedro Leopoldo, Minas Gerais, em 2003.

\section{MATERIAL E MÉTODOS}

O município de Pedro Leopoldo possui área de $293,21 \mathrm{~km}^{2}$ e está situado na região central de Minas Gerais. Localiza-se na região metropolitana de Belo Horizonte entre 4412'07' de longitude oeste e $19^{\circ} 37^{\prime} 00^{\prime}$ ' de latitude sul. Tem acesso a importantes rodovias, MG-424, MG-010 e MG-040. A sede da cidade está situada a $698 \mathrm{~m}$ de altitude. O clima é tropical úmido, com temperatura média anual de $22^{\circ} \mathrm{C}$, e o índice pluviométrico médio anual é de $1328,7 \mathrm{~mm} /$ ano. A população residente estimada para 2003 é de 57767 habitantes (IBGE, 2003), sendo $19,4 \%$ na área rural e $80,6 \%$ na área urbana.

Realizou-se inquérito soro-epidemiológico para a coleta de amostras de sangue de 2.185 cães, 
organizado em quatro grupos. Aplicou-se questionário semi-estruturado em cada residência visitada. Parte das informações também foi obtida por intermédio do Programa de Controle da Dengue, ocasião em que os agentes de saúde apontaram os registros referentes ao número de casas com cães e número de cães por casa. Para o processamento e a análise estatística, utilizou-se o Banco de Dados Epi Info 6.04b. O número mínimo de cães na amostra foi determinado aplicando-se a seguinte fórmula (Procedimientos...1979):

$\mathrm{n}=\frac{\mathrm{P}(100-\mathrm{P}) \alpha^{2}}{\left(\frac{\mathrm{Pxd}}{100}\right)^{2}}$ em que:

$\mathrm{n}=$ tamanho da amostra

$\mathrm{P}=$ prevalência esperada

$\alpha=$ grau de confiança $(\mathrm{P}<0,05)$

$\mathrm{d}=$ margem de erro esperada

$\mathrm{n}=\frac{3(100-3) 1,96^{2}}{\left(\frac{3 \times 25}{100}\right)^{2}}=1986$ cães

Para estimar a prevalência da LVC na área urbana, foram consideradas as áreas de zoneamento definidas pelo Programa Nacional de Controle da Dengue. Cada zona é composta de 800 a 1000 imóveis e separada de acordo com a homogeneidade física e a distância entre elas. Essa divisão permitiu padronizar o tamanho das áreas trabalhadas e considerar ambientes físicos semelhantes. A coleta foi realizada em contagem de 10 em 10 residências, sendo que, em todos os quarteirões, pelo menos um imóvel foi visitado, mesmo aqueles quarteirões que possuíam menos de 10 residências.

Foi coletado sangue de pelo menos um cão em cada domicílio, com idade acima de seis meses, dando-se preferência aos animais mais velhos. No grupo A, foram coletadas 1099 amostras na área urbana, no período de abril a julho, e 141 amostras na área rural, em setembro, totalizando 1240 cães. Nesse último, o inquérito foi feito em $100 \%$ das casas.

A coleta de amostras de sangue foi feita nas extremidades das orelhas com utilização de lanceta metálica descartável. A impressão do sangue foi feita sob papel de filtro Klabin n.25 (Controle..., 1999). Cães considerados suspeitos ao diagnóstico tiveram o sangue colhido novamente por punção venosa, após um período de 30 dias, para os assintomáticos e, após 15 dias, para os sintomáticos. Cães com resultado positivo tiveram sangue coletado imediatamente após, para confirmação do diagnóstico.

O teste de imunofluorescência indireta (RIFI) foi realizado com o antígeno $^{1}$ Leishmania amazonensis, amostra MHOM/BR/1960/BH6. O conjugado $^{2}$ utilizado na RIFI, antiimunoglobulina de cão, fração IgG marcada com isoticionato de fluoresceína. Os resultados foram interpretados como normatizados pelo Ministério da Saúde (Manual..., 2003), considerando-se como positivas as diluições de soro iguais ou maiores que 1:40. O teste imunoenzimático (ELISA) foi feito em placas de poliestireno de fundo chato com o mesmo antígeno da RIFI. A leitura da reação foi feita em leitor de ELISA $^{3}$ a $492 \mathrm{~nm}$. Os resultados foram expressos em valores de densidade óptica (DO). $O$ ponto de corte para o ELISA foi determinado a partir da média obtida valores dos cães controlenegativo, sendo multiplicada por 2. Em seguida, para aumentar a especificidade, multiplicou-se o resultado anterior por 1,2 .

A partir do inquérito, realizou-se atividade de bloqueio (grupo B) que consistiu na coleta de sangue de cães presentes em um raio de $200 \mathrm{~m}$ do foco positivo na área urbana e de $1 \mathrm{~km}$ na área rural, adotando-se a metodologia que é recomendada pelo Ministério da Saúde (Controle... 1999) para bloqueio de foco de casos humanos. Neste grupo, foram realizadas 656 coletas na zona urbana e 49 na zona rural.

No grupo C, foi realizada a coleta de sangue de 134 cães presentes durante a vacinação antirábica, os quais apresentavam pelo menos um sinal clínico característico da LVC. Os pontos de coleta foram escolhidos de acordo com o maior número de cães vacinados nas últimas vacinações anti-rábicas, sendo eles Centro, Lua e Lagoa de Santo Antônio.

No grupo (grupo D), foram colhidas amostras de sangue de 111 cães errantes, recolhidos pelo serviço de captura de pequenos animais do Controle de Zoonoses da Prefeitura de Pedro

\footnotetext{
${ }^{1}$ ICB-UFMG - Belo Horizonte, Brasil

${ }^{2}$ FIOCRUZ - Rio de Janeiro, Brasil

${ }^{3}$ Labsystems Multikan (version 4.0, 1994), Finlândia
} 
Leopoldo, entre os meses de abril a dezembro. As amostras foram submetidas ao teste ELISA. O destino destes cães foi a eutanásia ou o seu resgate pelo proprietário.

Os cães positivos nos grupos $\mathrm{A}, \mathrm{B}$ e $\mathrm{C}$ foram mapeados, utilizando-se como ferramenta o sistema de rádio-navegação mundial Global Positioning System (GPS), que fez o cálculo da localização espacial, em metros. Utilizou-se, para o georreferenciamento, o programa AUTOCAD 2000, tendo-se como base o mapa do município de Pedro Leopoldo, Projeto Mil, $2003^{4}$.

Os resultados das freqüências de cães positivos de acordo com a localização espacial foram analisados estatisticamente utilizando-se as provas de $\chi^{2}$ e o teste exato de Fisher (Sampaio, 1998). Fixou-se em $(\mathrm{P}<0,05)$ o nível de significância para rejeitar a hipótese de nulidade.

\section{RESULTADOS E DISCUSSÃO}

A prevalência de $1,4 \%$ de cães infectados para LVC no município não foi um resultado esperado (Tab. 1). Pedro Leopoldo está numa área endêmica e em expansão da LVC, como ocorre em Belo Horizonte, onde a prevalência foi de 5\% em 2002 e 8,5\% em 2003 (Resende, 2004). Dos sete municípios limítrofes com Pedro Leopoldo, seis possuem casos humanos e caninos notificados nos últimos dois anos, Ribeirão das Neves, Jaboticatubas, Esmeraldas, São José da Lapa, Matozinhos e Lagoa Santa (Silva et al., 2001). A presença de um eixo viário intenso com intercâmbio de animais de origem não local é outro fator de risco reconhecido para difusão da LVC. A ausência de notificação de casos humanos até o momento sugere a possibilidade de que a população canina apresente baixa prevalência para LV, uma vez que geralmente a enzootia canina tem precedido a ocorrência de casos humanos e a infecção em cães tem sido mais prevalente do que no homem (Brenner, 1957). Esta associação está descrita por Manual... (2003), que estima o aparecimento dos primeiros casos humanos em que as prevalências caninas sejam iguais ou acima de $2 \%$. Em Pedro Leopoldo, podese aceitar que a prevalência é baixa, e seriam recomendadas condutas priorizando a busca ativa

\footnotetext{
${ }^{4}$ IGA-SCTESMG - Belo Horizonte, Brasil

${ }^{5}$ RESENDE, S.M. Comunicação pessoal, Gerência de Controle de Zoonoses, SMS, Belo Horizonte, Brasil
}

de cães com suspeita clínica, educação em saúde, eutanásia de cães sororreagentes, vigilância e monitoramento.

Observou-se prevalência global de $1,4 \%$ de cães positivos no município, sendo as prevalências das áreas urbana e rural de $1,1 \%$ e 4,2\% respectivamente. Essa diferença foi significativa $(\mathrm{P}<0,001)$, e pode estar associada à entrada de cães positivos oriundos de localidades problemas para a enfermidade, à presença de $78 \%$ dos animais sem confinamento e/ou ao ambiente favorável à presença do vetor na área rural. Deane e Deane (1955) encontraram prevalências similares entre as áreas urbana e rural do município de Sobral, Ceará. Os autores ressaltaram que a prevalência esperada para a área urbana deveria ter sido maior, pois a presença de moradias contíguas e o estreito contato entre os cães é fator de disseminação da doença.

Verificaram-se nove zonas (40,9\%) com animais positivos no grupo A, e cinco no grupo B. Os cães positivos das zonas 2 e 16 eram referentes ao bloqueio das zonas 1 e 17, respectivamente, (Tab. 1). Este resultado demonstra que o combate à doença deve ser estratégico e priorizar as áreas de maior risco.

Dos 36 cães positivos ao bloqueio e ao inquérito, $47,2 \%$ eram machos e $52,8 \%$ fêmeas. Não foi observada diferença $(\mathrm{P}>0,05)$ em relação à distribuição da LVC por sexo, assim como observado por França-Silva et al. (2003), indicando que o sexo não é fator predisponente para infecção do cão em Pedro Leopoldo.

A idade estava concentrada principalmente entre um e 3,9 anos. Este fato não exclui a importância de outras faixas etárias tornarem-se infectadas. FrançaSilva et al. (2003), observaram que os cães, independente da idade, têm a mesma probabilidade de contrair LVC. Araújo et al. (2004), ao analisarem 530 prontuários de cães positivos da região metropolitana de Belo Horizonte, verificaram maior freqüência em cães de um a cinco anos, ao contrário de Alencar e Cunha (1963), que encontraram uma tendência crescente da infecção leishmaniótica do cão com o avanço da idade. 
Naveda et al.

Tabela 1 Prevalência da leishmaniose visceral canina no município de Pedro Leopoldo, Minas Gerais, 2003

\begin{tabular}{|c|c|c|c|c|c|c|c|c|c|}
\hline \multirow[b]{2}{*}{ Zona } & \multicolumn{3}{|c|}{ Cães positivos -grupo A } & \multicolumn{3}{|c|}{ Cães positivos -grupo B } & \multicolumn{3}{|c|}{ Total de cães positivos } \\
\hline & $\begin{array}{l}\text { Inquérito } \\
\text { amostral }\end{array}$ & $\%$ & $\begin{array}{l}\text { Amostra } \\
\text { coletada }\end{array}$ & $\begin{array}{c}\text { Bloqueio } \\
200 \mathrm{~m}\end{array}$ & $\%$ & $\begin{array}{l}\text { Amostra } \\
\text { coletada }\end{array}$ & Total & $\%$ & $\begin{array}{l}\text { Amostra } \\
\text { coletada }\end{array}$ \\
\hline 1 & 1 & 0,1 & 22 & 1 & 0,2 & 63 & 2 & 0,10 & 85 \\
\hline 2 & 0 & 0,0 & 50 & 1 & 0,2 & 47 & 1 & 0,06 & 97 \\
\hline 3 & 1 & 0,1 & 56 & 0 & 0,0 & 43 & 1 & 0,06 & 99 \\
\hline 4 & 0 & 0,0 & 37 & 0 & 0,0 & 6 & 0 & 0,00 & 43 \\
\hline 5 & 2 & 0,2 & 46 & 2 & 0,3 & 83 & 4 & 0,20 & 50 \\
\hline 6 & 0 & 0,0 & 43 & 0 & 0,0 & 0 & 0 & 0,00 & 43 \\
\hline 7 & 1 & 0,1 & 44 & 0 & 0,0 & 101 & 1 & 0,06 & 145 \\
\hline 8 & 0 & 0,0 & 28 & 0 & 0,0 & 15 & 0 & 0,00 & 43 \\
\hline 9 & 1 & 0,1 & 87 & 0 & 0,0 & 8 & 1 & 0,06 & 95 \\
\hline 10 & 0 & 0,0 & 31 & 0 & 0,0 & 20 & 0 & 0,00 & 51 \\
\hline 11 & 0 & 0,0 & 77 & 0 & 0,0 & 11 & 0 & 0,00 & 88 \\
\hline 12 & 2 & 0,1 & 107 & 4 & 0,6 & 82 & 6 & 0,30 & 189 \\
\hline 13 & 0 & 0,0 & 48 & 0 & 0,0 & 0 & 0 & 0,00 & 48 \\
\hline 14 & 0 & 0,0 & 45 & 0 & 0,0 & 0 & 0 & 0,00 & 45 \\
\hline 15 & 0 & 0,0 & 25 & 0 & 0,0 & 1 & 0 & 0,00 & 26 \\
\hline 16 & 0 & 0,0 & 40 & 2 & 0,3 & 0 & 2 & 0,10 & 40 \\
\hline 17 & 2 & 0,2 & 39 & 2 & 0,3 & 105 & 4 & 0,20 & 144 \\
\hline 18 & 0 & 0,0 & 82 & 0 & 0,0 & 0 & 0 & 0,00 & 82 \\
\hline 19 & 1 & 0,1 & 39 & 0 & 0,0 & 39 & 1 & 0,06 & 78 \\
\hline 20 & 0 & 0,0 & 23 & 0 & 0,0 & 6 & 0 & 0,00 & 29 \\
\hline 21 & 0 & 0,0 & 18 & 0 & 0,0 & 0 & 0 & 0,00 & 18 \\
\hline 22 & 1 & 0,1 & 110 & 1 & 0,2 & 16 & 2 & 0,10 & 126 \\
\hline ZU & 12 & 1,1 & 1099 & 13 & 1,9 & 656 & 25 & 1,4 & 1755 \\
\hline ZR & 6 & 4,2 & 141 & 5 & 10,2 & 49 & 11 & 5,9 & 190 \\
\hline Total & 18 & 1,4 & 1240 & 18 & 2,5 & 705 & 36 & 1,8 & 1945 \\
\hline
\end{tabular}

Observou-se que a origem do cão e seu deslocamento podem ter sido determinantes para a sua infeç̧ão. Na área rural, encontrou-se um cão procedente de Belo Horizonte, área endêmica em expansão. Na área urbana, a presença de cães de rua de Belo Horizonte, Sete Lagoas e Lagoa Santa e os deslocamentos entre esses municípios podem ter contribuído para disseminação da doença.

Foram encontrados três $(2,2 \%)$ cães positivos em 134 examinados no grupo $\mathrm{C}$, durante a campanha de vacinação anti-rábica. $\mathrm{O}$ valor de $2,2 \%$, comparado a $1,4 \%$ da amostragem obtida no grupo $\mathrm{A}$, não foi estatisticamente significativo $(\mathrm{P}<0,05)$. No grupo $\mathrm{C}$, todos os cães apresentavam sinais clínicos sugestivos, e a prevalência poderia ser maior. A pesquisa de Silva et al. (2001) revelou que a maior parte de cães positivos não apresentava qualquer alteração clínica. Caso o rastreamento fosse realizado exclusivamente com cães sintomáticos, provavelmente, não seria eficaz para estimar a real prevalência da LVC e, conseqüentemente, não poderia ser considerado para planejar as atividades de controle.

Todos os cães do grupo $\mathrm{C}$ tinham livre acesso à rua, e poderiam contribuir como os cães errantes, para disseminação da doença no município. Um deles era oriundo de Montes Claros, área endêmica para LV.

No grupo $\mathrm{D}$, quatro cães foram positivos $\mathrm{e}$ representaram 3,6\% de cães errantes positivos. A avaliação da prevalência neste grupo de animais pode contribuir para o monitoramento dos índices de LVC na localidade. O livre trânsito e a ausência de moradia possibilitam maior exposição aos vetores e a outros cães possivelmente infectados. Da mesma forma, podem representar potenciais fontes de 
disseminação. De acordo com os registros dos moradores, a respeito da origem dos cães, 1,4\% dizem que os cães foram recolhidos na rua, inclusive um cão positivo no grupo B. Existe consenso entre os profissionais que atuam na saúde pública, na maioria dos países, que cães soltos e vivendo nas ruas representam riscos efetivos de transmissão de doenças para o homem e, no caso da LVC, desde o início da década de 1950, Deane e Deane (1955) já alertavam que o cão é o principal reservatório e disseminador da LVC no meio rural e urbano.

A malha urbana do município está localizada em torno de importantes rodovias pavimentadas, e a sua configuração longitudinal, permite extenso contato com a área rural. $\mathrm{O}$ maior número de casos se concentra em torno da sede do município. Os cães positivos ao bloqueio evidenciam focos pontuais e importantes, em respectivas zonas, como observado nas zonas 5, 12 e 16.

\section{CONCLUSÕES}

A prevalência de LVC em cães, menor que $2 \%$, caracteriza o município de Pedro Leopoldo como área silenciosa, vulnerável e receptiva para a ocorrência de casos humanos com áreas de risco diferenciadas que demandam medidas sanitárias específicas. O conhecimento sobre a população canina permitirá estabelecer estratégias eficazes para as ações de controle no município e, para estimativa da prevalência de LVC, deverão ser considerados todos os cães com e sem sinais clínicos.

\section{REFERÊNCIAS BIBLIOGRÁFICAS}

BRASIL. Ministério da Saúde. Situação das doenças transmissíveis. Dispónível em $<$ www.saude.gov.br/svs/ Situação das doenças transmissíveis_> Acessado em: 30 de dezembro de 2004.

BRENER, Z. Calazar canino em Minas Gerais. 1957. 90f. Tese (Doutorado em Parasitologia) Faculdade de Medicina, Universidade Federal de Minas Gerais, Belo Horizonte.
CABRAL, A.L.V. Introdução e trajetória da leishmaniose visceral no município de Belo Horizonte, 1989 a 1994. 1995. 45f. Monografia (Especialização em Saúde Pública) - Escola de Saúde Pública de Minas Gerais, Belo Horizonte.

CONTROLE, diagnóstico e tratamento da leishmaniose visceral (Calazar). Brasília: Fundação Nacional da Saúde, 1999.

DEANE, L.M.; DEANE, M.P. Leishmaniose visceral urbana (no cão e no homem) em Sobral, Ceará. Hospital, v.47, p.75-87, 1955.

FRANÇA-SILVA, J.C. COSTA, R.T.; SIQUEIRA, A.M. et al. Epidemiology of canine visceral leishmaniosis in the endemic área of Montes Claros municipality, Minas Gerais state, Brazil. Vet. Parasitol., v.111, p.161-173, 2003.

INSTITUTO BRASILEIRO DE GEOGRAFIA E ESTATÍSTICA, 2003. Disponível em http:/www.ibge.gov.br Acessado em: 19 de julho de 2003.

MANUAL de Vigilância e Controle da Leishmaniose Visceral. Brasília: Ministério da Saúde, 2003. 120p.

MARTINS, A.V.; WILLIAMS, P.; FALCÂO, A.L. American sand flies (diptera: psychodidae, phlebotominae). Rio de Janeiro: Academia Brasileira de Ciências, 1978. 195p.

MINAS GERAIS. Secretaria de Estado da Saúde de Minas Gerais. Dados epidemiológicos Dispónível em <www. saúde.mg.gov.br/ Dados epidemiológicos > Acessado em: 30 de dezembro de 2004.

PROCEDIMIENTOS para estúdios de prevalência por muestreo. Buenos Aires: Centro Panamericano de Zoonosis, 1979. 35p. (Nota técnica n.128. rev.1)

SAMPAIO, I.B.M. Estatística aplicada à experimental animal. Belo Horizonte: Fundação de Ensino e Pesquisa em Medicina Veterinária e Zootecnia, 1998. 221p.

SILVA, E.S.; GONTIJO, C.M.F., PACHECO, R.S. et al. Visceral Leishmaniasis in the Metropolitan Region of Belo Horizonte, State of Minas Gerais Brasil. Mem. Inst. Oswaldo Cruz, v.96, p.285-291, 2001. 